\title{
The tuning-fork model of human social cognition: A critique
}

\author{
Pierre Jacob*
}

Institut Jean Nicod, UMR 8129, EHESS/ENS (Département d'études Cognitives)/CNRS, Ecole Normale Supérieure, Pavillon Jardin, 29, rue d'Ulm, 75005 Paris, France

\section{A R T I C L E I N F O}

\section{Article history:}

Received 20 December 2007

Available online $\mathrm{xxxx}$

\section{Keywords:}

Mirror neurons

Mindreading

Direct mapping

Action-understanding

Motor mirroring

Action-mirroring

Emotion-mirroring

Simulation

Imagination

Concept possession

\begin{abstract}
A B S T R A C T
The tuning-fork model of human social cognition, based on the discovery of mirror neurons (MNs) in the ventral premotor cortex of monkeys, involves the four following assumptions: (1) mirroring processes are processes of resonance or simulation. (2) They can be motor or non-motor. (3) Processes of motor mirroring (or action-mirroring), exemplified by the activity of MNs, constitute instances of third-person mindreading, whereby an observer represents the agent's intention. (4) Non-motor mirroring processes enable humans to represent others' emotions. After questioning all four assumptions, I point out that MNs in an observer's brain could not synchronically resonate with MNs in an agent's brain unless they discharged in a single brain in two distinct tasks at different times. Finally, I sketch a conceptualist alternative to the resonance model according to which a brain mechanism active in both the execution and the perception of e.g., the act of grasping is the neural basis of the concept of e.g., grasping.
\end{abstract}

(c) 2008 Published by Elsevier Inc.

\section{Introduction}

So-called "mirroring" processes are best exemplified by mirror neurons (MNs), i.e., sensorimotor neurons that fire both when an animal executes a transitive hand or mouth action (directed towards a target) and when the animal perceives the same kind of action being performed by a conspecific or by a human experimenter. MNs were first discovered (in the early 1990's) by single-cell recording in area F5 of the ventral premotor cortex (PMv) and later also in area PF of the inferior parietal lobule of monkeys. Subsequently, TMS and brain imaging experiments ${ }^{1}$ have been reported in support of the exis-

\footnotetext{
For comments on an earlier version of this paper, I am grateful to Patrick Cavanagh. For conversations on the topics of this paper, I am grateful to Jérôme Dokic, Gunther Knoblich, Hugo Mercier, Thomas Metzinger, Elisabeth Pacherie, Philippe Rochat, Natalie Sebanz, Mark Ashton Smith, Daniela Tagliafico, Iris Trinkler and Frédérique de Vignemont. A version of this paper was delivered at a Conference on "Self and other in social neuroscience and philosophy of mind" organized by Julian Kilverstein, Barry C. Smith and Tim Crane at the Institute of Philosophy, School of Advanced Study, at the University of London (in November 2007) at the APIC seminar at the Institut Jean Nicod (in December 2007) organized by Jérôme Dokic and Joëlle Proust and at a workshop at the university of Bilkent in Ankara (in April 2008) on "Taking the measure of mirror neurons" organized by Radu Bogdan. I am grateful to the organizers of these meetings and the audiences for their comments. I am also grateful to Alvin Goldman for email exchanges about the topic of this paper and for having sent me a copy of two recent papers of his on this topic. Finally, I am grateful to two anonymous referees for this journal and to the editor for their detailed critical comments.

* Fax: +33 144322699 .

E-mail address: Jacob@ehess.fr.

1 But, see Turella, Pierno, Tubaldi (2008) for a recent critical assessment of the brain-imaging evidence in favor of the existence of a mirror (neuron) system in humans.
} 
tence of a so-called mirror (neuron) system (MNS) or mirror circuit that responds to the execution and the perception of a wider range of actions, including both transitive and intransitive actions (not directed towards a target). ${ }^{2}$

MNs were so-called because their activity in an observer's brain is supposed to mirror (or resonate with) their activity in the agent's brain. Clearly, when MNs endogenously discharge in an agent's brain, their firing does not count as a mirroring process. Only the exogenously caused MN activity in an observer's brain, not the endogenously caused MN activity in an agent's brain, counts as a mirroring process.

After much discussion, no consensus has yet been reached about the exact nature of both the mechanism and the function of MN activity. Nonetheless, in 2004, Gallese, Keysers and Rizzolatti published a provocative paper entitled "A unifying view of the basis of social cognition", in which they offered a "unifying neural hypothesis on how individuals understand the actions and emotions of others". Their main claim was that "the fundamental mechanism at the basis of the experiential understanding of others' actions is the activation of the mirror neuron system. A similar mechanism, but involving viscero-motor centers, underlies the experiential understanding of the emotions of others". ${ }^{3}$ On their view, "the fundamental mechanism that allows us a direct experiential grasp of the mind of others is not conceptual reasoning but direct simulation of the observed events through the mirror mechanism. The novelty of our approach consists in providing for the first time a neurophysiological account of the experiential dimension of both action and emotion understanding". 4

On the basis of Gallese et al.'s (2004) use of the indefinite description "a direct experiential grasp of the mind of others", and borrowing a term from Saxe's (2005) reply to Goldman and Sebanz's (2005) reply to her original (2005a) critique of simulation theory, I will call Gallese et al.'s (2004) unifying view of social cognition the tuning-fork model of human social cognition. The tuning-fork model of human social cognition is a theory of human mindreading. Like most philosophers and psychologists, I assume that mindreading is the human cognitive ability to represent the psychological states (perceptions, emotions, intentions, desires, beliefs, etc.) of either self or others. ${ }^{5}$ Whereas a task of third-person mindreading consists in forming a belief about another's psychological state, a task of first-person mindreading consists in forming a belief about a psychological state of one's own.

What I call the tuning-fork model of human social cognition is a particular version of simulation-based approaches to human third-person mindreading. Like other versions of simulation-based approaches to third-person mindreading, it emphasizes the contribution of psychological and neural similarity to tasks of third-person mindreading. As Goldman (2006: 3536), a philosophical advocate of the simulation-based approach to mindreading, has emphasized, the English word "simulation" derives from the Latin verb simulare, which means "to duplicate" (or "to replicate"), and from the Latin adjective similis, which means "similar". What is distinctive of the tuning-fork model of human social cognition is that it is jointly committed to the four following assumptions.

(1) The first assumption is that mirroring processes, which underlie the so-called "experiential understanding" of another's action, are instances of mental simulation by virtue of being instances of motor simulation. (2) The second assumption is that mirroring processes can be motor or non-motor. (3) The third assumption is that processes of motor mirroring (action-mirroring), exemplified by the activity of MNs, constitute instances of third-person mindreading by virtue of which an observer can represent an agent's intention. (4) The fourth assumption is that non-motor mirroring processes enable humans to represent others' emotions. Assumption (2) presupposes that mirroring processes form a generic kind that can be divided into at least two species, the first of which underlies an observer's automatic response to another's action, and the second of which underlies an observer's automatic response to another's emotion. In light of assumption (2) of the tuning-fork model, in order not to beg the question against the possibility of emotion-mirroring, I will call "action-mirroring" or "motor mirroring" the first species of mirroring process, which is exemplified by MN activity in both the monkey's PMv and PF and by the human MNS.

This paper, which is a critical examination of the tuning-fork model of social cognition, is divided into three sections. In the first two sections, I restrict myself to action-mirroring. In the first section, I ask whether, in accordance with assumption (1), action-mirroring really qualifies as a species of motor simulation (or motor resonance). In Section 3, I examine the question of the link between action-mirroring and third-person mindreading. I first argue that, contrary to assumption (3), instances of action-mirroring could not constitute (in a strong sense) instances of third-person mindreading. I then examine possible causal links between tasks of mindreading and action-mirroring. In Section 4 , I argue that the enriched definition of a mirroring process required by the extension of action-mirroring into emotion-mirroring, in accordance with assumptions (2) and (4), faces insuperable difficulties. There, I offer an alternative conceptualist interpretation of some of the relevant evidence which serves as an empirical basis for the tuning-fork model of mindreading. The conclusion of my overall argument is that mirroring might turn out to be a by-product of third-person mindreading.

\footnotetext{
${ }^{2}$ I will use the abbreviation "MNs" to refer to mirror neurons and the abbreviated definite description "the MNS" to refer to the human mirror neuron system (or circuit).

3 Cf. Gallese, Keysers, and Rizzolatti (2004: 396).

4 Ibid.

5 On the view that an individual's psychological states (perceptions, emotions, etc.) are mental representations, a mindreader's belief about an individual's psychological states is a metarepresentation.
} 


\section{Is action-mirroring direct-matching?}

Following the discovery of MNs in area F5 of the ventral premotor cortex of monkeys, the prevailing view of action-mirroring has emphasized the congruency between the motor and the perceptual properties of MNs (and the MNS activity). In other words, it emphasizes the fact that MN activity is at work in both action execution and action observation. Assuming that the ability to execute a transitive action directed towards a physical target (as e.g., in grasping food) is necessary to the survival of primates, it seems as if no mechanism for understanding an observed transitive action performed by another could be more basic (or more primitive) than the very mechanism that would enable the observer (were he the agent) to execute the action in question. Thus, this emphasis on the congruency between the motor and the perceptual properties of MNs in the monkey PMv raises the intriguing prospect that a fairly elementary mechanism in the motor cognition of primates might constitute an instance of mental simulation, i.e., a process of motor simulation whereby the observer mentally rehearses (without executing) the agent's observed movements.

As Rizzolatti, Fogassi, and Gallese (2001) have stressed, depending on an observer's motor resources (or repertoire), there are perceived actions that can be mapped (or matched) onto the observer's motor repertoire and there are perceived actions that cannot be so mapped. Brain-imaging studies by Buccino et al. (2004) have revealed greater activations of motor (and premotor) areas when human observers see without hearing a video-clip displaying a human being produce silent speech or even a monkey produce silent lip-smacking than when they see a video-clip displaying a dog bark. In another brain-imaging study by Calvo-Merino, Glaser, Grezes, Passingham, and Haggard (2005), capoeira and ballet dancers saw short films displaying dance steps by either capoeira dancers or classical dancers. They found enhanced activations in the mirror systems of dancers observing movements from their own type of expert dancing. So perceptual responses to an agent's observed movements seem to reflect the observer's motor expertise and motor familiarity with the executed movements.

On this basis, Rizzolatti et al. (2001: 666) and Rizzolatti and Craighero (2004: 179) have drawn a contrast between two ways an action might be understood (or recognized): either trough a purely visual analysis of the agent's bodily movements or by means of the mapping of the agent's perceived movements onto the observer's motor repertoire. If and when an observer cannot map a perceived action onto his motor repertoire, then, according to Rizzolatti et al. (2001), it cannot be understood by what they call "motor resonance" (i.e., by mapping the agent's perceived movements onto the observer's motor repertoire): it can only be categorized on the basis of its visual properties. ${ }^{6}$ For example, since a primate cannot map a bird's wing movements onto its own motor repertoire, it can only categorize a bird's flight through a visual analysis.

Now, there is a weaker and a stronger version of the dual pathway thesis for action understanding. The weaker version is just the claim that perceived actions can be understood in two different ways. The stronger version, which deserves to be called the "direct-matching model of action-understanding", is the further claim, endorsed by Rizzolatti et al. (2001: 662 ), that "the results of the visual analysis of an observed action [...] are devoid of meaning [...]" and that motor involvement provides a distinctive kind of action understanding, which a purely visual analysis is unable to secure: "the main weakness of the visual hypothesis is that it does not indicate how the 'validation' of the meaning of the observed action is achieved" (Rizzolatti et al.'s, 2001: 666).

Clearly, the direct-matching model of action-understanding is a version of a simulation-based model of action-understanding. Action-mirroring is described as a process of motor simulation (or motor resonance) whereby the observer is caused by his perception of the agent's act to internally rehearse the agent's observed movements without executing them. By enabling the observer to map the agent's observed movements onto his own motor repertoire, motor mirroring is supposed to constitute a basic neural similarity between the agent and the observer whereby both entertain a shared motor representation of the action executed by the agent (cf. Vignemont \& Haggard, 2007). (Fig. 1) This neural similarity (or shared motor representation) is supposed to supply the observer with a special understanding of the meaning of the executed action, which a purely visual analysis is unable to provide. But this strong claim faces at least four putative problems. First, the very notion of a motor repertoire is not fully clear. Secondly, the evidence shows that the direct-matching model cannot be a complete model of action-understanding. Thirdly, MN activity has been reported for actions that the observer cannot execute. Finally, some recent evidence has led to a new model of chains of logically related MNs that is not consistent with the directmatching model.

\subsection{Motor or behavioral repertoire?}

Umiltà et al. (2001) have reported the significant finding that MNs in the ventral premotor cortex of monkeys fire when an observer sees an agent reach and grasp a target even if the target and the end of the agent's arm movement are hidden behind an occluder, but the monkey knows that there is a target behind the occluder because he has seen it prior to observing the agent's partly occluded act. On the other hand, the same MNs fail to discharge if the agent performs the same hand and arm movement but there is no target. Rizzolatti et al. (2001) and Rizzolatti and Craighero (2004) argue that this finding is evidence for the direct-matching model of action understanding: the firing of MNs is supposed to deliver a motor repre-

\footnotetext{
${ }^{6}$ As emphasized by an anonymous referee, application of "motor resonance" to MN activity is metaphorical talk. It is best explained, I think, by the idea that MN activity is motor simulation (without execution) of an observed act enabling the observer to map the agent's observed movements onto his own motor repertoire. If so, then the alleged resonance is likely to be a sub-personal, functional (not a personal) relation holding between the agent's and the observer's respective brain areas. This seems to fit Gallese's (2001: 45) construal of what he calls "the shared manifold of intersubjectivity".
} 


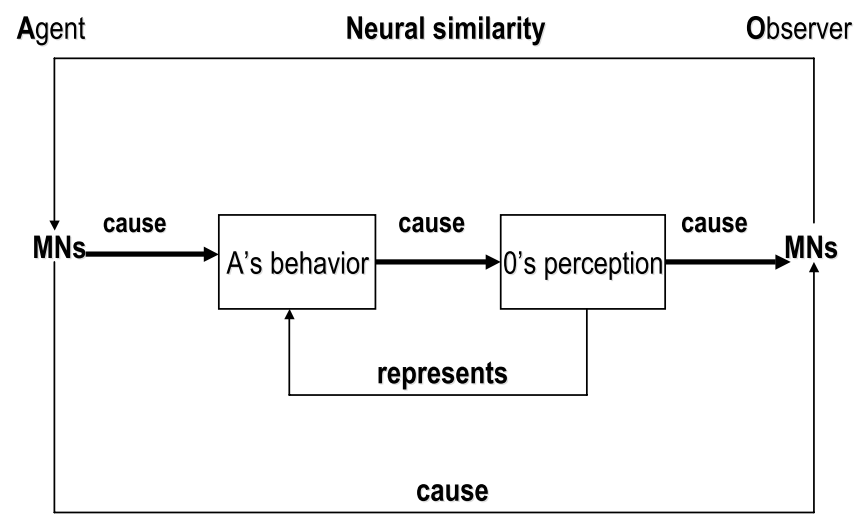

Fig. 1. Action-mirroring.

sentation of the "meaning" of the action. Since a mere pantomime of a reach-to-grasp action not directed towards a physical target lacks meaning in a non-human primate's mind, MNs don't fire when the animal perceives such a meaningless act.

Paradoxically, this finding, however, raises a problem for the direct-matching model of action-understanding. The problem is the following: the constitutive arm and hand movements involved in a reach-to-grasp act directed towards a target are demonstrably part of the monkey's motor repertoire. But the very same constitutive arm and hand movements involved in a reach-to-grasp act directed towards a target are also involved in a pantomime of the act of reach-to-grasp in the absence of a target. If so, then it seems as if the fact that MNs fail to fire when a monkey observes a pantomime of an act of reach-tograsp in the absence of a target should not be described as evidence for the view that action-mirroring consists in mapping an observed movement onto the observer's motor repertoire. Instead, it might be described as evidence for a revised version of the direct-matching model of action-understanding according to which action-mirroring consists in mapping an observed movement onto the observer's behavioral repertoire. But this formulation has the obvious drawback of turning the directmatching model into straight behaviorism. The question is: does action-mirroring generate action-understanding or does the former derive from the latter?

\subsection{MN activity for non-executable actions}

The first problem for the direct-matching model is that the fact that a bodily movement belongs to one's motor repertoire is not a sufficient condition for action-mirroring, let alone for action-understanding. The second problem arises from evidence that MN activity has been recorded in response to the perception of an act that the observer cannot execute. Ferrari, Rozzi, and Fogassi (2005) report a series of experiments in which they recorded MNs in area F5 of the ventral premotor cortex of monkeys after they had been exposed for a period of two months to the visual observation of human transitive actions made with tools such as picking up pieces of food with sticks and grasping them with pliers. They found MNs that fire both when the monkey executes some transitive hand and mouth actions and when the monkey observes actions performed by a human with a tool, which the monkey could not use. For example, they recorded one neuron that fires when the monkey grasps a small piece of food and when the monkey sees a human pick up a small piece of food with a sticker, but not when the human mimes the action with the sticker in the absence of food. They recorded another neuron that fires when the monkey grasps a piece of food with his hand and holds it. The same neuron also fires when the monkey sees a human agent grasp and hold the piece of food with a pair of pliers and when he sees the human pick up the piece of food and hold it with a sticker. The response of this neuron is weaker when the monkey sees the experimenter grasp the piece of food and hold it with his hand. These are clear instances of MNs that are active when the monkey sees actions that he cannot execute himself. So strictly speaking, these are MNs that fire even though the monkey is unable to map the perceived action onto his motor repertoire. This shows that the fact that an act belongs to one's motor or behavioral repertoire is not a necessary condition for action-mirroring. ${ }^{7}$

\subsection{Could direct-matching be a complete model of action-understanding?}

The third problem arises on the assumption that the direct-matching model provides a complete (or sufficient) model of action-understanding, not in need of any supplementation by a purely visual analysis of the observed action. Several years

\footnotetext{
${ }^{7}$ Furthermore, Wood, Glynn, Philips, and Hauser (2007a), Wood, Glynn, and Hauser (2007b) report behavioral evidence in non-human primates showing some (behavioral) understanding of human actions, e.g., throwing, that non-human primates cannot perform. Schubotz and Van Cramon (2004) report brainimaging evidence in humans showing activations in the human PMv for observation of static arrays of geometrical objects when subjects were requested to extract regularity of patterns. Costantini et al. (2005) report equally strong activation of the human PMv for the observation of bio-mechanically possible and impossible movements. Finally, Romani, Cesari, Urgesi, Facchini, and Aglioti (2005) report motor facilitation effects during the observation of bio-mechanically impossible finger movements.
} 
before MNs were discovered in area F5 of the ventral premotor cortex, Perrett and collaborators recorded cells in the monkey Superior Temporal Sulcus (STS), which are sensitive to a wider range of actions than MNs, including head- and eye-actions. However, unlike MNs, cells in the STS have purely perceptual properties: they never fire when the animal executes an action (see e.g., Keysers \& Perrett, 2004).

As Rizzolatti et al. (2001: 665) recognize, some of these cells in the STS fire when the monkey observes another agent perform a transitive act of reaching towards a target if the agent's gaze is directed towards his target, but they stop firing if the agent looks away during the act of reaching. Rizzolatti et al. (2001: 665-666) seem baffled by the complexity of the purely visual computations performed by cells in STS "that bind different types of visual features of an observed action": "these higher-order visual neurons combine the output of neurons that are specifically responsive to the observation of arm reaching with the output of neurons that are specifically responsive to the direction of attention, as conveyed by the direction of gaze". On the basis of the direct-matching model of action understanding, they are prepared to argue that "the sensory binding of different actions found in the STS is derived from the development of motor synergistic actions".

To put it mildly, it is unclear how, in accordance with the direct-matching model of action understanding, the purely perceptual activity of cells in the STS could derive from the motor resonance exemplified by MNs in the ventral premotor cortex for the observation of actions of reaching. As I understand it, what advocates of the direct-matching model of action understanding should demonstrate is that the sensitivity of cells in an observer's STS to an agent's gaze direction (or visual attention) depends on the preliminary activity of MNs which grounds the observer's understanding of (the meaning of) a perceived act of reaching. In other words, they should show that MNs in an observer's brain would fire when a monkey sees another agent perform a transitive act of reaching towards a target whether or not the agent looks away from his target during the act of reaching. Alternatively, they would have to show that the STS contains MNs (with both perceptual and motor properties) for gaze direction. Pending such discoveries, it seems more parsimonious to assume that the purely perceptual activity of cells in the STS responding to the agent's direction of gaze towards the putative target of his hand action is a precondition for MN activity in response to the perception of the transitive hand action.

Furthermore, there are good reasons why it is unlikely that there could be MNs for gaze direction. Consider two individuals $A$ and $B$ facing each other, engaged in verbal or non verbal communication and looking at each other's face. Suppose that suddenly, instead of staring at $B$ 's eyes, $A$ looks up above $B$ 's head. What will $B$ do? If $B$ 's eye-movements were governed by the activity of dedicated MNs, $B$ should mirror $A$ 's eye-movement and look above $A$ (just as $A$ looked above $B$ ). I predict that this is not what $B$ would do: I predict that $B$ would instead turn his head around and automatically look for the putative target of $A$ 's visual attention. So far the evidence suggests that MN activity in response to the perception of an act of reaching depends upon a prior purely visual analysis of the agent's direction of gaze towards the target. ${ }^{8}$

\subsection{The model of chains of logically related MNs}

In a series of elegant experiments in which a single motor act is embedded into two more complex actions, Fogassi et al. (2005) recorded MNs in the inferior parietal lobule (IPL) of monkeys during the execution and perception of acts of reachingto-grasp a target in order to either eat it or place it into a container. ${ }^{9}$ In the motor task, the container could be located either near the target or near the animal's mouth. Thus, one and the same act of grasping could be followed by either arm flexion (for eating or placing in a container near the mouth) or arm abduction (for placing near the target). In motor tasks, Fogassi et al. (2005) found that the activity of $65 \%$ of MNs that fire during execution of grasping is modulated by the more complex action of which it is a part. In particular, MNs fire selectively when grasping is part of placing, whether placing is near the target or near the mouth (and irrespective of the kinematic differences between arm abduction and arm flexion).

They also recorded MNs in IPL when the animal sees an experimenter grasp a piece of food in order to either eat it or place it into a container. They found that two thirds of recorded MNs fire selectively during observation of the act of grasping according to whether the grasping was for eating or for placing. Finally they found that $84 \%$ of MNs exhibit the same specificity in both motor and perceptual tasks. As they recognize, two factors may help the monkey to discriminate between grasping for eating and grasping for placing: one is whether or not the object grasped is food; and the other is whether or not a container is present in the context of the perceived action. Both are purely perceptual cues, whose processing as such would fail to elicit the activity of MNs. ${ }^{10}$

These experimental findings show that different MNs respond to the perception of one and the same act of grasping. On this basis, Fogassi et al. (2005) have endorsed a novel model of the activity of MNs, which, following Di Pellegrino, Fadiga, Fogassi, Gallese, and Rizzolatti (1992), they have called the "model of chains of logically related MNs". On this model (as

\footnotetext{
${ }^{8}$ Ricciardelli, Bricolo, Aglioti, and Chelazzi's (2002) experiments demonstrate the irresistible human tendency to follow and/or anticipate another's gaze and look where another is looking. By "imitating the oculomotor behavior" of another, they mean looking where the other is looking (not mimicking the other's eyemovement). They provide evidence for specialized mechanisms underlying joint attention, but they don't show the existence of action-mirroring for eyemovements.

${ }^{9}$ In one condition, the monkey brought the food to the mouth and ate it. In the other condition, the monkey was rewarded after correct accomplishment of the task of placing the target into a container (cf. Fogassi et al., 2005: 662).

${ }^{10}$ Cattaneo et al. (2007) used the same design as Fogassi et al. (2005) to examine the deficit of a population of young autistic children.
} 
emphasized by Rizzolatti \& Craighero, 2005: 115), when an observer sees an agent grasp a target within one of two distinct contexts, the MNs that fire in the observer's brain are not those that would govern the execution of the perceived act of grasping. Instead the MNs that fire in the observer's brain would govern the execution of the most likely act to follow the act of grasping as a function of contextual cues, e.g., bringing to the mouth or placing into a container. They cannot, therefore, be accommodated within the direct-matching (or motor resonance) model of MN activity based on the congruency between the perceptual and the motor properties of MNs. On the model of chains of logically related MNs, MN activity is now conceived as a predictive process, not as a process of motor resonance. In short: according to this new model, on the basis of the perceptual processing of contextual cues, MN activity is now conceived as a mechanism for predicting the agent's next motor act on the basis of perceptual cues. To resonate is to be receptive to a performed act. To predict is to anticipate an act that has not yet been performed. ${ }^{11}$

Thus, the evidence reported in this section is hard to reconcile with assumption (1) of the tuning-fork model, according to which MN activity (or action-mirroring) vindicates the strong version of the direct-matching model of action-understanding, i.e., that only mapping an agent's observed movements onto the observer's motor repertoire can supply "the meaning" of the action that a purely visual analysis fails to deliver. Instead, the evidence shows that, far from generating it, MN activity (or action-mirroring) might reflect or derive from action-understanding.

\section{What is the link between action-mirroring and mindreading?}

In 1998, Gallese and Goldman published an influential paper, which was the first to explicitly consider a possible link between action-mirroring and the simulation-based approach to third-person mindreading. In that paper, Gallese and Goldman (1998: 495) "explored [the] possibility [...] that MNs underlie the process of 'mindreading', or serve as precursors to such a process [... and that] MNs are part of [...] the folk psychologizing mechanism”. They further conjectured that "MNs represent a primitive version, or possibly a precursor in phylogeny, of a simulation heuristic that might underlie mindreading" (Gallese and Goldman, 1998: 498). More specifically, Gallese and Goldman (1998: 497) hypothesized that the "external" (or exogenous) activation of MNs might enable an observer to retrodict the agent's mental (or psychological) state (i.e., his intention) "by moving backwards from the observed action". On this view, exogenously caused MN activity would presumably take as input the perception of the agent's movements and retrodictively compute a representation of the agent's intention, i.e., the psychological cause of the agent's movements. ${ }^{12}$

Now, as Goldman (2008, in press) has recently pointed out, there are at least two different ways that action-mirroring could contribute to third-person mindreading: instances of the former could either constitute or cause instances of the latter.

\subsection{Why action-mirroring could not constitute third-person mindreading}

As I pointed out in the introduction, according to assumption (3) of the tuning-fork model of human social cognition, instances of action-mirroring are (or constitute) instances of third-person mindreading. As the paper by Gallese et al. (2004) clearly illustrates, there is a two-step slippery slope argument from the direct-matching model of action-understanding to the thesis that instances of action-mirroring constitute instances of third-person mindreading. According to the directmatching model of action-understanding, unlike a purely visual hypothesis, only action-mirroring can supply "the meaning" of a perceived action. First, it is a small step from the claim that only action-mirroring can supply "the meaning" of a perceived act to the claim that action-mirroring offers an "experiential understanding of others' actions" (Gallese et al., 2004: 396). Secondly, it is tempting to move from the latter claim to the further claim that action-mirroring is "the fundamental mechanism that allows a direct experiential grasp of the mind of others" (Gallese et al., 2004). I take it that Gallese et al. (2004) are hereby endorsing the view that tasks of third-person mindreading could be achieved by action-mirroring.

In an experiment described in the previous section, Fogassi et al. (2005) found that different MNs in the monkey IPL fire in response to the perception of a single act of grasping embedded within two distinct more complex actions, one of which was eating and the other of which was placing food into a container. They interpreted the result of their experiment as evidence that chains of logically related MNs in the monkey IPL "not only code the motor act but also allow the observer to understand the agent's intentions" (Fogassi et al. 2005: 662). If so, then chains of logically related MNs in the monkey IPL can be said to perform tasks of third-person mindreading (the constitutive interpretation of the link between mirroring and mindreading).

Arguably, as recognized by Jeannerod (2006), it is controversial whether monkeys do represent and attribute intentions to conspecifics. ${ }^{13}$ But lacoboni et al. (2005) did conduct a brain-imaging study in humans with the same design as the single-cell recording study conducted by Fogassi et al. (2005) in monkeys. They showed healthy human adults a single motor act embedded into two more complex actions by means of pairs of films divided into three conditions. In the Context condition, subjects saw objects (a tea-pot, a mug, cookies, etc.) arranged as if either before tea (the "drinking" Context) or after tea (the "cleaning" Context), but no action. In the Action condition, subjects saw a human hand grasp a mug using either a precision grip or a

\footnotetext{
11 For a detailed analysis, see Jacob (2008).

12 Note that it is unlikely that by mentally rehearsing an agent's perceived movement, an observer could mentally represent its psychological cause. Cf. Jacob (2008).

13 According to Fogassi et al. (2005: 662), in the executive task, monkeys were trained to place food into a container by being "rewarded after correct accomplishment of the task". So the selective discharge of MNs in the perceptual task may reflect associative learning instead of spontaneous mindreading.
} 
whole-hand prehension, with no contextual elements present. In the Action condition, subjects saw either one or the other of the two acts of prehension embedded in either the drinking Context (Intention to drink condition) or the cleaning Context (Intention to clean condition). In other words, in perceiving one or the other of the single acts constitutive of the Action condition embedded into two different Contexts in the Intention condition, the observer could represent it as part of two distinct complex actions. The perception of the Context condition cannot trigger MN activity since in this condition, there are affordances, but there is no action to be seen. ${ }^{14}$ Viewing the Intention condition led to a significantly stronger activation in the right inferior frontal areas (believed to be rich in MNs) than viewing the Action condition (which in turn was stronger than viewing the Context condition without any action).

Iacoboni et al. (2005: 529) take their experiment to demonstrate that "premotor mirror neuron areas-areas active during the execution and the observation of an action-previously thought to be involved only in action recognition are actually also involved in understanding the intentions of others". If so, then the human MNS could be said to perform tasks of third-person mindreading. Given the tension between the simulation-based direct-matching model of action-mirroring and the new model of chains of logically related MNs, the question whether action-mirroring could constitute third-person mindreading should be examined separately for each model. In fact, both the Fogassi et al. (2005) study and the Iacoboni et al. (2005) study leave it open whether they advocate the replacement of the simulation-based direct-matching model of action-mirroring by the new predictive model of chains of logically related MNs or the coexistence of the two models. ${ }^{15}$

As I stated in the introduction, to perform a task of third-person mindreading it to form a belief about another's psychological state $S$ (e.g., another's intention). I further assume that one could not form a belief about another's psychological state $S$ (e.g., another's intention) unless one possessed and applied to the agent the concept of $S$ (e.g., the concept intention). ${ }^{16}$

Let us first consider the simulation-based direct-matching model of action-mirroring. On this model, as a result of actionmirroring exogenously triggered by the perception of a motor act of grasping a mug, an observer could at best form the intention to grasp a mug without executing the act. Clearly, to form the intention to grasp a mug (without executing the act) is not the same psychological state as believing of an agent (distinct from self) that she intends to grasp a mug. Given the above definition of mindreading, only the latter, not the former, constitutes an instance of third-person mindreading. Furthermore, whereas one can form the intention to grasp a mug and lack the concept intention, one cannot believe that another intends to grasp a mug (and thus ascribe to him the intention) unless one possesses the concept intention. If so, then action-mirroring falls short of third-person mindreading.

Now consider the new model of chains of logically related MNs. Fogassi et al. (2005: 666) write that chains of logically related MNs "allow the monkey to predict what is the goal of the observed action and, thus, to 'read' the intention of the acting individual". Similarly, according to Iacoboni et al. (2005: 535), "to ascribe an intention is to infer a forthcoming new goal, and this is an operation that the motor system does automatically". However, as Goldman (in press: pp. 4-5 of manuscript) insightfully notices, the notion of a goal is equivocal: it may refer to a physical target (e.g., a mug), to an act to be performed on a physical target (e.g., the agent's bringing the mug to his mouth) and to an agent's intention (e.g., the agent's prior intention to drink). Now, unlike both the target and the agent's act, only the agent's prior intention to drink is a genuine mental representation (of a possible, non-actual state of affairs): it is a psychological cause of the agent's perceived act of grasping the mug. ${ }^{17}$

On the model of chains of logically related MNs, the perception of an act of grasping a mug triggers the prediction of the next most likely motor act given contextual cues (e.g., the agent's bringing the mug to his mouth). On this model, actionmirroring takes as input a perceived act of grasping and the output it delivers is a motor representation of another act (the agent's bringing the mug to his mouth). On this model, action-mirroring could not deliver a representation of the agent's prior intention to drink, because action-mirroring is supposed to be a predictive device. Given that the agent's prior intention to drink is the cause of the agent's perceived act, action-mirroring could not predict the cause of the agent's perceived act from the perception of the act (i.e., the effect of the intention). It could only retrodict the agent's prior intention to drink from the perception of the act; what it can predict is the agent's next most likely act. ${ }^{18}$ Thus, on the model of chains of logically related MNs, action-mirroring also falls short of third-person mindreading.

\subsection{Does action-mirroring cause third-person mindreading?}

Goldman (2006, 2008, in press), one of the most eloquent advocates of the simulation approach to human mindreading over the past 20 years, agrees that action-mirroring cannot constitute third-person mindreading. He endorses instead the weaker thesis that the latter causally depends on the former. As Borg (2007) and Jacob (2008) have noted, unless one sub-

\footnotetext{
14 Affordances can trigger the discharge of so-called “canonical” neurons, not of MNs. Cf. Rizzolatti, Fogassi, and Gallese (2000).

15 Note that on Gallese and Goldman's (1998) view, the agent's intention is retrodictively computed by action-mirroring from the perception of his movements. Goldman (2008c): (pp. 6-7 of manuscript) recognizes the tension between the two models, but he espouses the latter view and concludes from the studies that there are two kinds of mirror neurons.

${ }^{16}$ I italicize the names of concepts.

17 Following Searle's (1983), Pacherie's (2000) and others' terminology, it is natural to characterize the agent's intention to drink as his prior intention because it is chronologically and causally prior to both the agent's acts of grasping the mug and bringing it to the mouth. Cf. Jacob (2008).

18 If one knows an agent's beliefs and desires, then arguably one can predict an agent's prior intention. For example, knowing that an agent both wants to read and believes that there is not enough light, one could presumably predict that the agent will form the prior intention to turn the light on. But clearly such a prediction is beyond the mechanism of action-mirroring.
} 


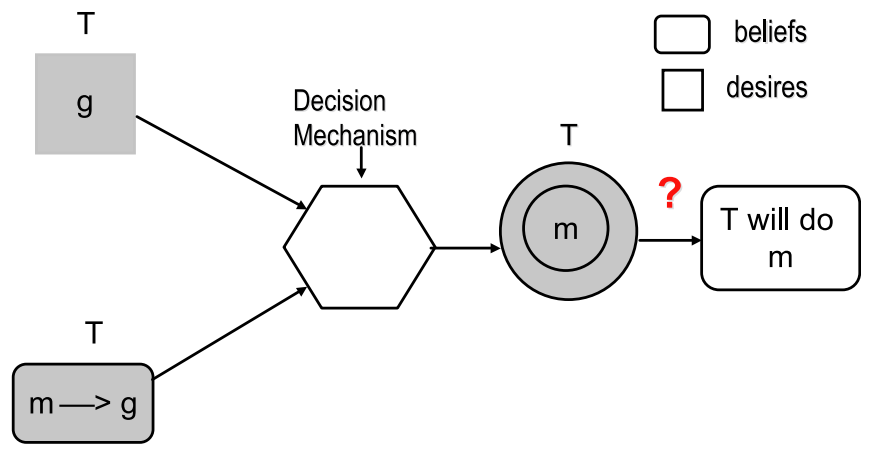

Fig. 2. Simulation-based account of the prediction of another's decision (from Gallese \& Goldman, 1998). Squares stand for goals or desires. Oval shapes stand for beliefs. Grey areas stand for the interpreter's pretend states. White areas stand for the interpreter's own states.

scribed to behaviorism, one would not be tempted to endorse the view that the perception and mental rehearsal of an agent's motor act is a sufficient basis for forming a reliable belief about the agent's prior intention. Although the weaker thesis asserting a causal dependency between third-person mindreading and action-mirroring sounds far more plausible than the stronger thesis of a constitutive link, the weaker thesis faces at least two problems: the problem of the teleological stance and the problem of the gap in the causal link.

\subsection{The teleological stance problem}

Violation-of-expectation studies by Gergely, Nadasdy, Csibra, and Biro (1995), Csibra, Biró, Koós, and Gergely (2003) and Gergely and Csibra (2003) have demonstrated that human infants as young as 12-month old both automatically interpret others' actions as goal-directed and expect agents to perform the most efficient act given the physical constraints. ${ }^{19}$ For example, 12-month-olds, who have been habituated to seeing a 2D circle jump over a 2D rectangular obstacle on a computer screen in a parabolic trajectory, are more surprised (i.e., look longer) if they see the circle jump again once the obstacle has been removed rather than if they see the circle move in a straight line (i.e., exemplify a novel trajectory that they have never seen before). According to Gergely and Csibra (2003), these experimental results show that 12-month-olds ascribe goals and apply what they call the "teleological stance" to others' actions.

Goldman (2006, 2008, in press), who subscribes to a simulation-based approach to third-person mindreading, primarily thinks of action-mirroring as an instance of an exogenous process of motor simulation that automatically creates a neural similarity between an agent and a observer. Goldman (2006) further claims that action-mirroring causally contributes to what he calls "low-level" tasks of third-person mindreading. Tasks of low-level mindreading are "comparatively simple, primitive, automatic and largely below the level of consciousness" (Goldman, 2006: 113). ${ }^{20}$ Now given Gergely and Csibra (2003) findings, the adoption of the teleological stance by 12-month-olds seems to be early evidence of low-level mindreading activity of goal-ascription. ${ }^{21}$ The problem for Goldman's causal claim is that 12 -month-olds cannot jump, let alone fly, over obstacles. ${ }^{22}$ So as argued by Jacob and Jeannerod (2005) the Gergely and Csibra findings strongly suggest that action-mirroring (i.e., mapping an agent's observed movement onto one's motor repertoire) cannot be a necessary condition for a 12-month-old human infant to ascribe a goal to an agent. But if so, then not all instances of low-level mindreading can be causally dependent upon action-mirroring. ${ }^{23}$

\subsection{The gap in the causal link}

As Goldman (in press: p. 2 of manuscript) indicates, the causal link interpretation between action-mirroring and low-level third-person mindreading is a special case of the general claim that mental simulation causally contributes to tasks of thirdperson mindreading. The thesis of the causal dependency of third-person mindreading upon either action-mirroring or men-

\footnotetext{
19 "Goal-directed" does not mean directed towards a physical target, but guided by a mentally represented aim.

20 The contrast between low-level and high-level tasks of third-person mindreading might be drawn in terms of the amount of inhibition required: higher-level mindreading requires more inhibition that lower-level mindreading. For example, passing a standard false belief task requires inhibiting one's own correct representation of the location of the object in order to represent another's incorrect belief.

${ }^{21}$ In light of Onishi and Baillargeon's (2005) and Surian, Caldi, and Sperber's (2007) evidence for the representation of others' false beliefs by respectively 15month-olds and 13-month-olds, this does not seem like an unreasonable proposal Cf. Leslie (2005).

22 Goldman (2008c: p. 13 of manuscript) challenges my claim that the Gergely and Csibra findings provide evidence for low-level mindreading of goalascription. But there he refers to Kuhlemeier, Wynn, and Bloom's (2003) finding that 12-month-olds exhibit a preference for 'helping' over 'hindering' motions performed by geometrical stimuli, which I earlier cited (cf. Jacob, 2008). I could agree with Goldman that a preference for helping over hindering "does not clearly attest to mindreading". But I would maintain that the Gergely and Csibra findings about goal-ascription are evidence of low-level mindreading in Goldman's sense.

${ }^{23}$ But see section 3 for Goldman's response and my rebuttal.
} 
tal simulation in general leaves a crucial gap unfilled within the putative causal chain. On the simulation-based directmatching model, an instance of action-mirroring exogenously triggered by the perception of a motor act of grasping a mug could at best lead an observer to form the intention to grasp the mug without executing the act. The question is: how is the observer's tokening of an intention to grasp the mug turned into the observer's belief that the agent intends to grasp the mug?

Consider now the standard simulation-based account of the ascription (or prediction) of another's decision (Fig. 2). Call the other $T$ for target. The interpreter creates in his own mind a pretend goal (or desire) $g$ and a pretend instrumental belief to the effect that producing action $m$ is an efficient means of achieving goal $g$, where both the pretend goal $g$ and the pretend belief that producing action $m$ is an efficient means of achieving goal $g$ are supposed to be relevantly similar to $T$ s actual goal and instrumental belief. Then the interpreter feeds the pretend goal and the pretend belief into his own decision-making mechanism, which in turn outputs a pretend decision, e.g., the pretend decision to do $m$. Unsurprisingly, the standard simulation-based account of decision-ascription now faces the very same question that is faced by action-mirroring: how is the pretend decision to do $m$ transformed into the interpreter's belief (or prediction) that target $T$ will decide to do $m$ ?

What is distinctive of simulation-based approaches to mindreading is the appeal to interpersonal neural and/or psychological similarity. Further appeal to interpersonal similarity in and by itself will not solve this question. The response to both versions of the same question is that the output of either action-mirroring or pretence is being embedded into a higher-order (or metarepresentational) belief. Arguably, action-mirroring or pretence may enable a mindreader to mimick (or duplicate) another's psychological state. However, to perform a full task of third-person mindreading is to form a genuine (higher-order) belief about another's psychological state. Only by breaking out of the (hermeneutical) circle of interpersonal similarity and by shifting up to metarepresentational ascent can the questions be answered. ${ }^{24}$

\subsection{An alternative causal link}

Now Goldman (in press: p. 2 of manuscript) only considers two ways action-mirroring might relate to third-person mindreading: instances of the former might constitute instances of the latter or instances of the latter might causally depend on instances of the former. But Goldman's dichotomy is not exclusive: there is a third alternative according to which understanding (or representing) an agent's prior intention causally enhances action-mirroring. On this alternative view, human mindreading is achieved by specialized metarepresentational mechanisms that take as input the perception of contextual cues and action-mirroring is causally enhanced by third-person mindreading. As I said, it is controversial whether the perceptual task investigated by Fogassi et al. (2005) requires monkeys to perform tasks of third-person mindreading. By contrast, in the perceptual task investigated by Iacoboni et al.'s (2005), it is natural to assume that human observers do represent the agent's prior intention to either drink or clean. ${ }^{25}$ The brain-imaging evidence from Iacoboni et al.'s (2005) study is consistent with the hypothesis that subjects might form a perceptual representation of the agent's prior intention to e.g., drink, based on the perception of contextual cues. Furthermore, the enhanced MN activity might itself result from the observer's perceptually formed representation of the agent's prior intention to drink.

Action-mirroring generates a motor representation of an agent's act. According to the novel model of chains of logically related MNs, in response to the perception of a given motor act, on the basis of contextual cues, MNs predict the agent's next most likely act. Computational theorists of action make a useful distinction between two kinds of "internal models" of action: forward and inverse models. ${ }^{26}$ Forward models take as input a representation of an agent's motor command and compute the sensory consequences of the agent's action. Inverse models take as input a representation of the agent's goal (or prior intention) and compute the best motor command required to satisfy the agent's goal. As Rizzolatti et al. (2001) and Rizzolatti and Craighero (2004) have emphasized, the fact that MN activity generates motor representations of actions does not make action-mirroring suitable for computing the sensory consequences of actions. Instead it makes them suitable for computing motor commands. Now, suppose that an observer can form a perceptual representation of an agent's prior intention on the basis of contextual cues. If so, then, as Csibra (2005) and I (in Jacob, 2008) have argued, action-mirroring takes as input a perceptually based representation of the agent's prior intention and, in accordance with inverse models of action, it computes a representation of the motor commands that would best enable the agent to achieve his prior intention. If so, then action-mirroring causally depends on third-person mindreading, and not vice-versa. ${ }^{27}$

\footnotetext{
${ }^{24}$ Similarly, action-mirroring, pretence or imagination may enable one to engage into a game of make-believe required to appreciate a work of fiction. But there is a gap between engaging in such a game and forming metarepresentational beliefs (and stating facts) about or describing a work of fiction.

25 Iacoboni et al. (2005) report that, when explicitly probed, their subjects did verbally report the agent's prior intention to drink upon seeing the Intention to drink condition and his prior intention to clean upon seeing the Intention to clean condition.

26 Cf. Wolpert (1997), Wolpert and Ghahramani (2000), Wolpert, Ghahramani, and Flanagan (2001).

27 The results of the brain-imaging experiment reported by Brass, Schmitt, Spengler, and Gergely (2007) are consistent with this view: Brass et al. (2007) report more activity in observers' brain areas known to be involved in third-person mindreading for the observation of an agent's knee movements when they are unexpected as opposed to expected. The results obtained in the perceptual task in the experiment on autistic children reported by Cattaneo et al. (2007) also are consistent with this view. For an assessment of the hypothesis that autism arises from a deficit in the human MNS, cf. Southgate, Gergely, and Csibra (2007).
} 


\section{Mirroring, imagining and concept-possession}

Goldman (2006, 2008, in press) does not subscribe to the tuning-fork model of social cognition because, although he endorses the view that third-person mindreading causally depends on action-mirroring, he nonetheless rejects the view that the latter constitutes the former. However, Goldman (2006, 2008, in press) accepts assumptions (2) and (4) of the tuningfork model, namely that there are at least two species of mirroring processes: motor mirroring (or action-mirroring) and non-motor mirroring (e.g., emotion-mirroring). As Goldman (in press: p. 1 of manuscript) puts it, "the best current evidence for mirror-based mindreading is not found in the motoric domain but in the domains of emotion and sensation". ${ }^{28}$

\subsection{A sample of the putative evidence for non-motor mirroring}

Of the rich putative evidence for mirroring outside the motoric domain, I shall selectively concentrate on experimental work in both healthy and brain-lesioned human subjects showing that the same brain areas are active when someone experiences some basic emotion and also when she perceives the face of an individual experiencing the same basic emotion. ${ }^{29}$ Brain-imaging experiments performed by Wicker et al. (2003) show that the anterior part of the insula and to a lesser extent the anterior part of the cingulate cortex (neither of which is part of the human MNS involved in action-mirroring) are active both when a human subject experiences disgust (as a result of inhaling some unpleasant smell) and when he sees a video-clip displaying the facial expression of another person experiencing disgust. On this basis, Wicker et al. (2003) have drawn an analogy between action-mirroring (i.e., the MNS underlying the execution and the perception of actions) and a putative mirror system coupling the first-person experience of disgust and the third-personal observation of the facial expressions of others experiencing disgust.

As emphasized by Goldman (2006: 132), one of the questions raised by this brain-imaging study is whether subjects who were observing video-clips displaying the faces of others experiencing disgust were attributing disgust to individuals whose faces they were observing (i.e., whether they were performing a third-person task of mindreading). Paired deficits in both the experience of disgust and the facial recognition of disgust in brain-lesioned human patients would seem to provide evidence for the view that, in healthy subjects, the brain areas whose activity is necessary for experiencing disgust are also necessary for ascribing disgust to others. For example, patient NK studied by Calder, Keane, Manes, Antoun, and Young (2000) suffered damage jointly to the insula and basal ganglia. In response to explicit questionnaires, NK displayed a selective impairment in the first-personal experience of disgust (whereas his experience of anger and fear was in the normal range). NK also showed selective impairment in disgust-recognition when viewing the facial expression of others while they were experiencing disgust. Thus NK's lesion deprived him jointly of the first-personal experience of disgust and the ability to recognize disgust in others.

Prima facie, the brain-imaging study conducted by Wicker et al. (2003) and the neuropsychological investigation of brainlesioned human patient NK by Calder et al. (2000) may seem to support the view that in healthy subjects, the recognition of others' disgust (and the attribution of disgust to others) is achieved by a mirroring process. But on the one hand, it is not altogether clear whether, given the evidence, one's representation of another's disgust results from a distinctive mirror neuron circuit for disgust or from connecting the action-mirroring system (i.e., the human MNS for the execution and perception of actions) to the insula and cingulate cortex. On the latter option, the underlying mirroring process would consist of actionmirroring whereby the observer would map the target's facial expressive movement onto his own repertoire of facial movements. On the other hand, if the recognition of disgust is achieved by a distinctive process of emotional mirroring, dissociable from action-mirroring, then presumably when a healthy subject ascribes disgust to another via the perception of the other's face, she should undergo a first-personal experience of disgust. It is at least questionable whether recognition of another's disgust via the perception of his face involves (in all cases) the observer's first-personal experience of disgust. ${ }^{30}$

Instead of pursuing the detailed investigation of such subtle empirical issues, I now want to consider the broad theoretical questions raised by extending the definition of mirroring processes from action-mirroring to emotion-mirroring.

\subsection{Could there be non-motor mirroring?}

When MNs were first discovered in the premotor cortex of monkeys, the fundamental intuition underlying the implicit definition of action-mirroring was that MN activity in the agent's brain causes a similar MN activity in an observer's brain by means of a three-step causal chain. First, MN activity in the agent's brain generates the agent's bodily movement (or behav-

\footnotetext{
28 Thus, Goldman (2008b: 27) sharply disagrees with Hurley's (2008) own attempt at elaborating and refining the scope of action-mirroring by means of "control theory" (and internal models of action), which restricts itself to the execution and perception of action, and cannot be extended to the mirroring of emotions. Gallese (2001: 46) also has expressed the view that "the discovery of MNs in the monkey PMv has unveiled a neural mechanism, that, in light of more recent findings, appears to be present in a variety of non-motor related human brain structures".

29 Goldman and Sripada (2005) and Goldman (2006, ch. 6, 2008a, 2008c) contain helpful and detailed discussions of this evidence.

30 Unless the mirroring of another's disgust in an observer's insula and cingulate cortex is correlated with the inhibition of the observer's own first-personal experience of disgust. Similarly, the fact that patients with Mobius syndrome, whose facial muscles are paralyzed, are able to recognize others' disgust also raises other subtle empirical issues that I cannot deal with in this paper. One basic question is whether muscular paralysis is inconsistent with motor simulation. For some relevant discussion cf. Goldman and Sripada (2005) and Goldman (2006, ch. 6).
} 
ior). Secondly, the observer perceives (visually or otherwise) the agent's bodily movement. Thirdly, the observer's perception of the agent's motor act causes MN activity in his brain (as indicated in Fig. 1). ${ }^{31}$ Unlike a motor act, a basic emotional experience (of e.g., disgust) is an internal unobservable psychological state. It may, but it need not, give rise to any observable behavioral effect (e.g., a facial expression). As philosophical critics of logical behaviorism taught us long ago, what Putnam (1963) called "super-Spartans" or "super-Stoics" might experience pain or disgust and inhibit any behavioral expression. If so, then there is a missing link in the causal chain linking the activity of the relevant brain area underlying one individual's experience of disgust to the activity of the same brain area in an observer for the latter to mirror the former. Pain or disgust themselves are not observable; only the "normal" behavioral effects of pain or disgust are. But if the "normal" behavioral effects of pain or disgust are inhibited (e.g., for Spartan reasons), then the causal chain necessary for emotional mirroring is incomplete. So the question is: how could action-mirroring be enriched and extended into an observer's automatic response to another's emotional state?

Goldman (2008) offers a response to this challenge in two steps. First, he internalizes the definition of an endogenous activation of MNs (or the MNS). Secondly, he liberalizes the definition of a mirroring process (or exogenous activation of MNs or the MNS) by introducing the notion of a sign. On the classical intuitive definition of action-mirroring (illustrated in Fig. 1), the endogenous activation of MNs was taken to underlie the overt execution of a motor act. On Goldman's modified definition, an endogenous activation of MNs is said to underlie an internal "mental or cognitive event". According to Goldman (2008) extended definition, a mirroring process (or event) is not elicited merely by the observer's perception of the agent's overt act. Instead, it is elicited by the observer's detection of a "sign that another individual undergoes or is about to undergo the same type of mental or cognitive event".

Now Goldman's extended definition faces, I think, the following dilemma: either the new definition of mirroring as the detection of a sign (that a target undergoes some internal mental event) is restricted to a natural sign in Grice's (1957) sense or it is not. ${ }^{32}$ If it is, then it runs the risk of turning third-person emotional mindreading into mere emotional contagion. If it is not, then it runs the risk of not being compatible with Goldman's own $(2004,2006)$ important distinction between two different kinds of mental simulation: mirroring (which is an exogenously caused process) and pretence or imagination (which is an endogenous process), as illustrated in Fig. 3:

Gallese, 2003 and Gallese et al.'s (2004) endorsement of the third assumption of the tuning-fork model amounts to accepting the first horn of the dilemma. Gallese et al. (2004) emphatically deny the conceptual character of the experiential understanding of another's emotion generated by a mirroring process. For example, they (Gallese et al., 2004: 396) write: "there are neural mechanisms (mirror mechanisms) that allow us to directly understand the meanings of actions and emotions of others by internally replicating [...] them without any explicit reflective mediation. Conceptual reasoning is not necessary for this understanding". Thus, they seem to embrace the view that emotion-mirroring is really a process of emotional contagion-not a process of emotional understanding, let alone a process whose output is third-person emotional mindreading (i.e., an observer's belief that another is experiencing an emotion).

Arguably, the collapse of third-person emotional mindreading onto emotional contagion reflects an underlying behaviorist construal of the content of emotional experiences themselves. I earlier mentioned the fact that a super-Spartan might inhibit any behavioral expression of disgust (or pain). Conversely, for the fun of pretence, a human being may also exhibit the "normal" behavioral expression of disgust (or pain) while failing to experience the relevant emotion. So the normal behavioral expression of a basic emotion is neither a necessary nor a sufficient condition of the emotional experience itself.

On several occasions, Goldman seems ready to embrace the second horn of the dilemma. For example, Goldman (to appear: p. 5 of manuscript) endorses the view that an observer's response to a "good imitation of a pained expression" counts as a genuine mirroring process even though "the imitation is not a genuine expression, or (natural) sign, of an endogenous mirror event". ${ }^{33}$ With Goldman (in press: p. 2 of the manuscript), consider a film featuring a virtual human-like artifact (or robot). Since the artifact has no brain of its own, it thereby lacks beliefs, intentions and emotions. If and when it moves, its movements do not count as actions caused and monitored by its intentions since it does not have any. Now, by endorsing the view that an observer's perceptual responses to a film displaying the artifact's movements and behavioral expressions of pretend (or pseudo-) emotions count as a genuine mirroring process, he again embraces the second horn of the dilemma. Finally, when Goldman (in press: p. 13 of manuscript) is willing to count the perceptual responses of 12-month-olds to the motions of geometrical stimuli in Kuhlemeier et al. (2003) violation-of-expectation study as instances of action-mirroring, he also embraces the second horn of the dilemma. Given that geometrical stimuli lack a nervous system, they could not have goals, intentions or emotions. Nor could their motions count as goal-directed actions that 12-month-olds could mirror.

\footnotetext{
${ }^{31}$ As I have already argued in Section 3, this intuitive definition of action-mirroring had to be modified into the novel model of chains of logically related MNs in order to accommodate data designed to establish that action-mirroring constitutes mindreading, in accordance with assumption (3) of the tuning-fork model.

32 In Grice's (1957) sense, whereas the word "fire" non-naturally means fire, smoke is a natural sign of fire because instances of the latter cause instances of the former.

${ }^{33}$ I have added "natural": an imitation of a pained expression cannot be a natural sign of pain.
} 


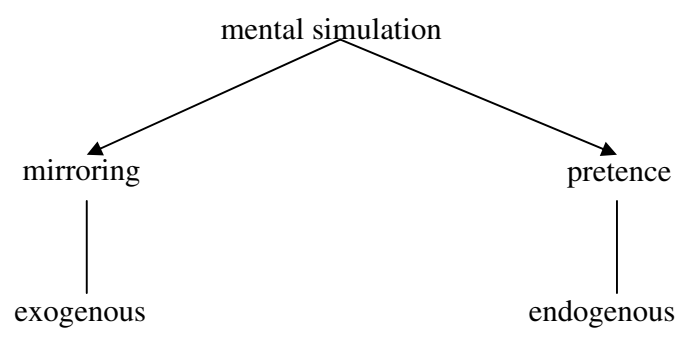

Fig. 3.

As I see it, the problem with embracing the second horn of the dilemma is that it is not easy to reconcile with Goldman's (2004, 2006) own distinction between mirroring and imagination (or pretence). On Goldman's simulation-theoretic view, both mirroring and pretence (or imagination) are processes that generate a relevant similarity between an interpreter (or observer) and a target. But there are important differences between pretence or imagination and mirroring. Action-mirroring is an exogenous process whereby the activity of some brain mechanism in individual $B$ is caused by $B$ 's perception of another individual $A$ 's observable behavior, which in turn is caused by the activity of some relevantly similar brain mechanism in $A$. By transitivity of the causal relation, the activity of $B$ 's brain mechanism turns out to be caused by the activity of $A$ 's relevantly similar brain mechanism (see Fig. 1). This, I take it, is the main reason why Gallese et al. (2004) assume that the mirroring process underlying the so-called "experiential" understanding of another's emotion is mediated by the activity of viscero-motor centers. ${ }^{34}$ This is also why I cannot agree with Goldman's (2006: 211) claim that "the assumption that all mirroring phenomena are motor phenomena [...] is unwarranted".

Whereas action-mirroring involves the exogenously caused perception of another's act, imagining (or pretending) is an endogenous process. Whereas visual perception takes retinal inputs, visual imagery (or visualizing) takes inputs from memory. Arguably, unlike perceiving, imagining (in either the imagistic or the propositional sense) is a mental action: whereas seeing is something that happens to one, forming a mental image is something one does. ${ }^{35}$ Unlike perceiving, imagining is under the control of the will: one can willfully decide to form a mental image. Arguably, one can also willfully decide to attend and look at objects; but one cannot willfully decide to see visible objects present in one's visual field. ${ }^{36}$ Now, if mirroring processes are not restricted to motor mirroring (or action-mirroring), then the risk is that it becomes impossible to sort out the kind of interpersonal psychological similarity generated, respectively, by a mirroring process and by either the imagination or some more complex inferential (or reasoning) process, neither of which should qualify as a mirroring process. I shall give two examples.

First, suppose that whereas I really enjoy inhaling smell $S$, you find inhaling $S$ really disgusting and I know it. Suppose that, while you do inhale $S$ and you experience disgust, I now imagine, with my eyes closed, what it is for you to experience disgust upon inhaling $S$. Suppose further that your insula gets activated as a result of your experience of disgust and my insula gets activated as a result of my vividly imagining your experience of disgust. According to the view that all mirroring is action-mirroring (or motor mirroring), activity in my insula could not count as mirroring the activity of your insula unless the former was caused by my seeing the facial behavioral effect of your experience of disgust.

Secondly, suppose that $B$ believes that someone is in pain as a result of her belief that she $(B)$ is in pain, which results from her experience of pain. Suppose further that $B$ utters the sentence "I'm in pain" in $A$ 's presence. Suppose that upon perceiving and understanding $B$ 's utterance and via $A$ 's representation of $B$ 's communicative intention, $A$ forms the belief that $B$ wants him to believe that she believes that she is in pain. Suppose that as a result of this belief, $A$ comes to believe that $B$ is in pain. Suppose finally that from his belief that $B$ is in pain, $A$ infers that someone is in pain. Both $A$ and $B$ now share the belief that someone is in pain. Consider the inferential transition from the belief that $B$ is in pain to the belief that someone is in pain. This reasoning, which may be realized by the same neural process $N$ in both $A$ 's and $B$ 's brains, is a psychological process shared by $A$ and $B$. $B$ 's utterance of "I'm in pain" was caused by $B$ 's belief that she is in pain and it caused $A$ 's belief that $B$ is in pain. The belief that $B$ is in pain caused both $A$ and $B$ to acquire the belief that someone is in pain. A token of neural process of type $N$ in $B$ 's brain caused another token of neural process of type $N$ in $A$ 's brain via $A$ 's understanding of $B$ 's utterance.

\footnotetext{
${ }^{34}$ This insistence on viscero-motor centers is hard to reconcile with Gallese's $(2001,2004)$ claim that mirroring should not be restricted to action-mirroring (or motor related structures).

35 Advocates of the so-called "enactive" conception of perception, such as Noë (2004), deny this and claim that perception also is something one does. But, see Jacob (2006) for a rebuttal.

${ }^{36}$ For a valuable discussion of the distinction between imagining and perceiving, cf. McGinn (2004).
} 
If mirroring were not confined to motor mirroring (or action-mirroring), then in the first example, we could say that the endogenous activation of my insula as a result of my imagining your experience of disgust is an instance of mirroring. But surely this would be inconsistent with Goldman's (2006) distinction between mirroring and imagination. In the second example, we surely don't want to say that some mirroring process mapped the inferential transition from $B$ 's brain activation of $N$ to A's brain activation of $N$. Nor do we want to say that the inferential transition in A's brain is the output of a mirroring process that took the inferential transition in $B$ 's brain as an input. But if mirroring is not restricted to motor mirroring, then why not say that the inferential transition in B's brain was mapped onto a similar inferential transition in A's brain via some mirroring process?

\subsection{A conceptualist alternative}

Since MNs were first recorded in the ventral premotor cortex of monkeys, the prevalent interpretation of the experimental findings has been cast in terms of a process of interpersonal simulation whose output is a neural and/or psychological interpersonal resonance or similarity. Clearly, however, MN activity could not achieve interpersonal resonance or similarity across the brains of two distinct individuals at a single time unless MNs fired in a single brain on different occasions. ${ }^{37}$ Now, as Sperber (2004) has suggested, a brain mechanism that is active within a single brain in both the execution of a transitive act (e.g., grasping) and in the perception of the same act performed by a conspecific looks very much like a neural mechanism underlying the concept of the act in question (i.e., grasping). ${ }^{38}$

Arguably, the conceptual representation of the act of grasping is an abstract representation that brackets the difference between the purely motor representation and the purely visual representation of the act. The view that MN activity is the neural basis of the concept of such a specific act as grasping would make sense of the variations in the statistical congruency between the motor and the perceptual properties of MNs noted by Csibra $(2005,2007)$. Whereas a significant proportion of MNs are generally reported as being "strictly congruent", a majority is said to be "broadly congruent". In fact, on the basis of Rizzolatti et al.'s (2000; Rizzolatti, Fogassi, \& Gallese, 2004) notion of a motor vocabulary, what I now am calling a conceptual representation of the act of grasping is just what Rizzolatti et al. (2001) and Rizzolatti and Craighero (2004) call the motor representation commonly entertained by an agent and an observer.

If a conceptual representation of grasping brackets the distinction between executive instances and perceptual representations of the act, then possession of the concept of grasping does not require $100 \%$ statistical congruency between the MNs that fire during respectively the execution and the perception of an act of grasping. Furthermore, the view that MN activity is the neural basis of some motor acts further helps resolve the tension between the direct-matching model and the model of chains of logically related MNs. If some MN activity is the neural basis of the concept of grasping, then it does not underlie third-person mindreading. But it makes sense that the application of the concept of grasping triggered by the perception of an act of grasping would inferentially give rise to the related concept of e.g., drinking. Conversely, it also fits with the view according to which a representation of an agent's prior intention to e.g., drink (derived from contextual cues) should trigger the application of the concept of grasping.

Nor does it follow from the conceptualist approach that there is no interesting difference between an agent and an observer. ${ }^{39}$ For instance, it is entirely consistent with the conceptualist approach that, unlike an observer, an agent has available information about his being the author of the act by means of an efference copy of his motor instruction. Insofar as the efference copy of an agent's motor instruction serves as the basis of the sense of agency, the conceptualist approach can accommodate the phenomenological difference between being an agent and being an observer of an act performed by another.

Similarly, the fact that the same brain areas (the insula and part of the cingulate cortex) are active both when one experiences disgust and when one sees the facial expression of a disgusted individual also looks like good evidence for the claim that these brain areas are the neural bases for the conceptual representation of disgust. Now unlike the concept of grasping, the concept of disgust is the concept of a psychological state (cf. Vignemont \& Singer, 2006). So the brain areas that underlie the conceptual representation of the act of grasping are not directly involved in third-person mindreading. But the brain areas that underlie the conceptual representation of disgust (on the basis of face-based emotion recognition) are involved in what Goldman (2006: ch. 6) calls "low-level" mindreading.

Simulation-based approaches to third-person mindreading have long emphasized the crucial role of psychological similarity between a target (or agent) and an interpreter. The discovery of MNs has been interpreted as the discovery of a vindication of the simulation approach to mindreading by showing that the existence of a neural mechanism for motor simulation in the primate brain, which would create a neural similarity between an agent and an observer and would enable the latter to make sense of an agent's action from the perception of the agent's movements. Similarly, the results of brainimaging experiments on humans showing that the same brain areas are active when an individual experiences disgust and when he sees the facial expression of another who experiences disgust have been interpreted as demonstrating the neural

\footnotetext{
${ }^{37}$ As suggested by an anonymous referee, when an agent sees his own hand movement, MNs might fire in a single brain in a dual task (of action-execution and action-perception). If so, then it would not be strictly correct to say that MNs fire in a single brain in different tasks at different times. But it is difficult to test the hypothesis that the firing of MNs in an agent's brain also underlies the agent's visual perception of his own act.

${ }^{38}$ On this view, the discovery of MNs in the ventral premotor cortex of monkeys might be said to vindicate the application of Fodor's (1975) hypothesis of a language of thought composed of concepts (or cross-modal mental symbols) to the cognition of non-human primates (who lack a language faculty).

39 The question was raised by an anonymous referee.
} 
similarity between the two individuals. On the conceptualist alternative, the neural (or psychological) similarity (or resonance) between two individuals is a side-effect or a by-product of the existence of neural mechanisms within a single brain underlying the possession of relevant concepts.

\section{Conclusions}

The cognitive neuroscientists who discovered MNs have espoused the tuning-fork model of human social cognition and heavily emphasized the resonating role of MNs (and the MNS) in human social cognition. As I pointed out in the last section of this paper, two individual brains could not resonate at a single time unless there was a mechanism in a single brain that was active in two different tasks (e.g., a motor task and a perceptual task) at two different times. As I further pointed out, a brain mechanism that is active in both the execution of a transitive act (e.g., grasping) and in the perception of the same act performed by a conspecific looks very much like a neural mechanism underlying the concept of the act in question.

Arguably, natural selection may have favored neural mechanisms active in a single primate brain at different times because such mechanisms underlie the possession of the relevant concept (of e.g., grasping). For example, possession of the concept of grasping in a primate brain might be relevant in a highly competitive social context for making precise predictions about others' behavior related to e.g., food. If so, then action-mirroring (or motor resonance) is an evolutionary by-product (or side-effect), not the direct result of natural selection.

Two human brains could not empathize at a single time unless a single brain had neural mechanisms active in two different tasks (e.g., the first-person experience of disgust and the recognition of others' disgust). The fact that the same brain areas are active in a human brain when an individual experiences disgust and when he or she sees the face of another who is experiencing disgust also suggests that these brain areas constitute the neural basis of the concept of disgust. Arguably, natural selection may have favored individuals with brain structures underlying the concept of disgust because it is highly advantageous for such omnivorous creatures as humans to be able to detect potentially poisonous food from recognizing others' experience of disgust. If so, then the emotional resonance whereby an observer empathizes with the experience of another's disgust might also be an evolutionary by-product, not the direct result of natural selection.

\section{References}

Borg, E. (2007). If mirror neurons are the answer, what was the question? Journal of Consciousness Studies, $14,5-19$.

Brass, M., Schmitt, R. M., Spengler, S., \& Gergely, G. (2007). Investigating action understanding: Inferential processes versus action simulation. Current Biology, 17(24), 2117-2121.

Buccino, G., Lui, F., Canessa, N., Patteri, I., Lagravinese, G., Benuzzi, F., et al (2004). Neural circuits involved in the recognition of actions performed by nonconspecifics: An fMRI study. Journal of Cognitive Neuroscience, 16, 114-126.

Calder, A. J., Keane, J., Manes, F., Antoun, N., \& Young, A. W. (2000). Impaired recognition and experience of disgust following brain injury. Nature Neuroscience, 3, 1077-1078.

Calvo-Merino, B., Glaser, D. E., Grezes, J., Passingham, R. E., \& Haggard, P. (2005). Action observation and acquired motor skills: An fMRI study with expert dancers. Cerebral Cortex, 15, 1243-1249.

Cattaneo, L., Fabbri-Destro, M., Boria, S., Pieraccini, C., Monti, A., Cossu, G., et al (2007). Impairment of actions chains in autism and its possible role in intention understanding. Proceedings of the National Academy of Sciences of the United States of America, 104(45), 17825-17830.

Costantini, M., Galati, G., Ferretti, A., Caulo, M., Tartaro, A., Romani, G. L., et al (2005). Neural systems underlying observation of humanly impossible movements. Cerebral Cortex, 15, 1761-1767.

Csibra, G. (2005). Mirror neurons or emulator neurons? Available from: http://mirrorneurons.free.fr.

Csibra, G. (2007). Action mirroring and action understanding. In P. Haggard, Y. Rossetti, \& M. Kawato (Eds.), Sensorimotor foundations of higher cognition. Attention and performance XXII. Oxford: Oxford University Press.

Csibra, G., Biró, S., Koós, O., \& Gergely, G. (2003). One-year-old infants use teleological representations of actions productively. Cognitive Science, 27, $111-133$.

Di Pellegrino, G., Fadiga, L., Fogassi, L., Gallese, V., \& Rizzolatti, G. (1992). Understanding motor events: A neurophysiological study. Experimental Brain Research, 91, 176-180.

Ferrari, P. F., Rozzi, S., \& Fogassi, L. (2005). Mirror neurons responding to observation of actions made with tools in monkey ventral premotor cortex. European Journal of Neuroscience, 17, 1703-1714.

Fodor, J. A. (1975). The language of thought. New York: Crowell.

Fogassi, L., Ferrari, P. F., Gesierich, B., Rozzi, S., Chersi, F., \& Rizzolatti, G. (2005). Parietal lobe: From action organization to intention understanding. Science, 308, 662-667.

Gallese, V. (2001). The 'shared manifold' hypothesis. Journal of Consciousness Studies, 8(5-7), 33-50.

Gallese, V. (2003). The manifold nature of interpersonal relations: The quest for a common mechanism. Philosophical Transactions of the Royal Society of London Series B: Biological Sciences, 358, 517-528.

Gallese, V., Keysers, C., \& Rizzolatti, G. (2004). A unifying view of social cognition. Trends in Cognitive Sciences, 8, $396-403$.

Gallese, V., \& Goldman, A. (1998). Mirror neurons and the simulation theory of mindreading. Trends in Cognitive Sciences, $12,493-501$.

Gergely, G., \& Csibra, G. (2003). Teleological reasoning about actions: The naïve theory of rational actions. Trends in Cognitive Sciences, 7, 287-292.

Gergely, G., Nadasdy, Z., Csibra, G., \& Biro, S. (1995). Taking the intentional stance at 12 months of age. Cognition, 56, $165-193$.

Goldman, A. (2004). Mirror systems. Social understanding and social cognition. Available from: http://www.interdisciplines.org/mirror/paper/3.

Goldman, A. (2006). Simulating minds, the philosophy, psychology and neuroscience of mindreading. Oxford: Oxford University Press.

Goldman, A. (2008). Mirroring, mindreading and simulation. In Pineda, J.A. (Ed.), Mirror neuron system: The role of mirroring processes in social cognition. Humana Press.

Goldman, A. I. (2008). Does one size fit all? Hurley on shared circuits. Behavioral and Brain Sciences, 31(1), $27-28$.

Goldman, A. (in press). Jacob on mirroring, simulating and mindreading. Mind and Language.

Goldman, A., \& Sebanz, N. (2005). Simulation, mirroring, and a different argument from error. Trends in Cognitive Sciences., 9, 320.

Goldman, A., \& Sripada, C. (2005). Simulationist models of face-based emotion recognition. Cognition, 94, 193-213.

Grice, H. Paul (1957). Meaning. In H.P. Grice (1989). Studies in the way of words, Cambridge, Mass: Harvard University.

Hurley, S. (2008). The shared circuit model: How control, mirroring and simulation can enable imitation, deliberation and mindreading. Behavioral and Brain Sciences, 31, 1-58. 
Iacoboni, M., Molnar-Szakacs, I., Gallese, V., Buccino, G., Mazziotta, J. C., \& Rizzolatti, G. (2005). Grasping the intentions of others with one's own mirror neuron system. PLoS Biology, 3, 529-535.

Jacob, P. (2006). Why visual experience is likely to resist being enacted. Psyche, 12(1)http://www.psyche.cs.monash.edu.au.

Jacob, P. (2008). What do mirror neurons contribute to human social cognition? Mind and Language, 23(2), 190-223.

Jacob, P., \& Jeannerod, M. (2005). The motor theory of social cognition: A critique. Trends in Cognitive Sciences, 9(1), 21-25.

Jeannerod, M. (2006). Motor cognition. Oxford: Oxford University Press.

Keysers, C., \& Perrett, D. I. (2004). Demystifying social cognition: A Hebbian perspective. Trends in Cognitive Sciences, 8(11), $501-507$.

Kuhlemeier, V., Wynn, K., \& Bloom, P. (2003). Attribution of dispositional states by 12 month-olds. Psychological Science, $14,402-408$.

Leslie, A. (2005). Developmental parallels in understanding minds and bodies. Trends in Cognitive Sciences, 9(10), 459-462.

McGinn, C. (2004). Mindsight, image, dream, meaning. Cambridge, Mass.: Harvard University Press.

Noë, A. (2004). Action in perception. Cambridge, Mass: MIT Press.

Onishi, K. H., \& Baillargeon, R. (2005). Do 15-month-old infants understand false beliefs? Science, 308, 255-258. April 2005.

Pacherie, E. (2000). The content of intentions. Mind and Language, 15(4), 400-432.

Putnam, H. (1963). Brains and behavior. In Putnam, H. (1975). Philosophical papers (Vol. 2), Mind, language and reality. Cambridge: Cambridge University Press.

Ricciardelli, P., Bricolo, E., Aglioti, S. M., \& Chelazzi, L. (2002). My eyes want to look where your eyes are looking: Exploring the tendency to imitate another individual's gaze. Neuroreport, 13(17), 2259-2264.

Rizzolatti, G., Fogassi, L., \& Gallese, V. (2000). Cortical mechanisms subserving object grasping and action recognition: A new view on the cortical motor functions. In M. S. Gazzaniga (Ed.), The new cognitive neurosciences. Cambridge, Mass: MIT Press.

Rizzolatti, G., Fogassi, L., \& Gallese, V. (2001). Neurophysiological mechanisms underlying the understanding and imitation of action. Nature Review of Neuroscience, 2, 661-670.

Rizzolatti, G., Fogassi, L., \& Gallese, V. (2004). Cortical mechanisms subserving object grasping, action understanding, and imitation. In M. S. Gazzaniga (Ed.), The cognitive neurosciences III. Cambridge, Mass: MIT Press.

Rizzolatti, G., \& Craighero, L. (2004). The mirror-neuron system. Annual Review of Neuroscience., 27, $169-192$.

Rizzolatti, G., \& Craighero, L. (2005). Mirror neurons: A neurological approach to empathy. In J.-P. Changeux, A. R. Damasio, W. Singer, \& Y. Christen (Eds.), Neurobiology of human values. Berlin: Springer.

Romani, M., Cesari, P., Urgesi, C., Facchini, S., \& Aglioti, S. M. (2005). Motor facilitation of the human cortical-spino system during observation of biomechanically. NeuroImage, 26, 755-763.

Saxe, R. (2005a). Against simulation. Trends in Cognitive Sciences, 9(4), 174-179.

Saxe, R. (2005b). Tuning forks in the mind: Reply to Goldman and Sebanz. Trends in Cognitive Sciences, 9, 321.

Schubotz, R., \& Van Cramon, Y. (2004). Sequences of abstract nonbiological stimuli share ventral premotor cortex with action observation and imagery. The Journal of Neuroscience, 24(24), 5467-5474.

Searle, J. (1983). Intentionality. An essay in the philosophy of mind. Cambridge: Cambridge University press.

Southgate, V., Gergely, G., Csibra, G., 2007. Does the mirror neuron system and its impairment explain human imitation and autism? In Pineda, J.A. (Ed.), Mirror neuron system: The role of mirroring processes in social cognition, Humana Press.

Sperber, D. (2004). "Mirror neurons" or “concept neurons"? Available from: http://www.interdisciplines.org/mirror/1.

Surian, L., Caldi, S., \& Sperber, D. (2007). Attribution of beliefs by 13-month-old infants. Psychological Science, 18(7), 580-586.

Turella, L., Pierno, A.C., Tubaldi, F., \& Castiello, U. (2008) Mirror neurons in humans: Consisting of confounding evidence?. Brain and Language, in press, doi:10.1016/j.bandl.2007.11.002.

Umiltà, M. A., Kohler, E., Gallese, V., Fogassi, L., Fadiga, L., Keysers, C., et al (2001). I know what you are doing': A neurophysiological study. Neuron, 32, 91-101.

Vignemont, F., \& Singer, T. (2006). The empathic brain: How, when and why? Trends in Cognitive Sciences, 10, $435-441$.

Vignemont, F. \& Haggard, P. (2007). Action execution and action observation: What is shared?

Wicker, B., Keysers, C., Plailly, J., Royet, J.-P., Gallese, V., \& Rizzolatti, G. (2003). Both of us disgusted in my insula: The common neural basis of seeing and feeling disgust. Neuron, 40, 655-664.

Wolpert, D. M. (1997). Computational approaches to motor control. Trends in Cognitive Sciences, 1, $209-216$.

Wolpert, D. M., \& Ghahramani, Z. (2000). Computational principles of movement neuroscience. Nature Neuroscience, 3, $1212-1217$.

Wolpert, D. M., Ghahramani, Z., \& Flanagan, J. R. (2001). Perspectives and problems in motor learning. Trends in Cognitive Sciences, 5, $487-494$.

Wood, J., Glynn, D. D., Philips, B. C., \& Hauser, M. (2007a). The perception of rational, goal-directed action in non-human primates. Science, 317, 1404-1405.

Wood, J. N., Glynn, D. D., \& Hauser, M. D. (2007b). The uniquely human capacity to throw evolved from a non-throwing primate: An evolutionary dissociation between action and perception. Biology Letters, 3(4), 360-364. 